\title{
The chromosome-level Hemerocallis citrina Borani genome provides new insights into the rutin biosynthesis and the lack of colchicine
}

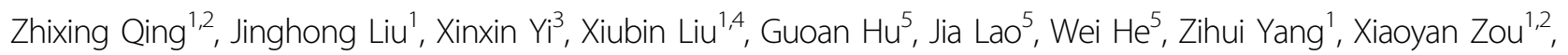
Mengshan Sun ${ }^{1}$, Peng Huang ${ }^{1,4}$ and Jianguo Zeng ${ }^{1,2,6}$

\begin{abstract}
Hemerocallis citrina Borani (huang hua cai in Chinese) is an important horticultural crop whose flower buds are widely consumed as a delicious vegetable in Asia. Here we assembled a high-quality reference genome of $H$. citrina using single-molecule sequencing and $\mathrm{Hi}-\mathrm{C}$ technologies. The genome assembly was $3.77 \mathrm{~Gb}$ and consisted of 3183 contigs with a contig N50 of $2.09 \mathrm{Mb}$, which were further clustered into 11 pseudochromosomes. A larger portion (3.25 Gb or 86.20\%) was annotated as a repetitive content and 54,295 protein-coding genes were annotated in the genome. Genome evolution analysis showed that $\mathrm{H}$. citrina experienced a recent whole-genome duplication (WGD) event at $\sim 15.73$ million years ago (Mya), which was the main factor leading to many multiple copies of orthologous genes. We used this reference genome to predict 20 genes involved in the rutin biosynthesis pathway. Moreover, our metabolomics data revealed neither colchicine nor its precursors in $\mathrm{H}$. citrina, challenging the long-standing belief that this alkaloid causes poisoning by the plant. The results of our disruptive research are further substantiated by our genomic finding that $\mathrm{H}$. citrina does not contain any genes involved in colchicine biosynthesis. The high-quality genome lays a solid foundation for genetic research and molecular breeding of $\mathrm{H}$. citrina.
\end{abstract}

\section{Introduction}

Hemerocallis citrina Borani is a perennial crop and its flower buds are one of the most commonly consumed vegetables in Asia. This plant has been widely grown in Asian countries, including China, Japan, and Korea, and has also been regarded as the traditional mother's flower in Chinese culture for a thousand years ${ }^{1,2}$. H. citrina flower buds have been used to relieve depression and promote lactation, as documented in the medicinal book "Compendium of Materia Medica," which is a famous

\footnotetext{
Correspondence: Peng Huang (huangpeng@hunau.edu.cn) or Jianguo Zeng (zengjianguo@hunau.edu.cn)

${ }^{1}$ Hunan Key Laboratory of Traditional Chinese Veterinary Medicine, Hunan Agricultural University, Changsha, Hunan 410128, China

${ }^{2}$ College of Veterinary Medicine, Hunan Agricultural University, Changsha, Hunan 410128, China

Full list of author information is available at the end of the article

These authors contributed equally: Zhixing Qing, Jinghong Liu, Xinxin Yi,

Xiubin Liu
}

Chinese encyclopedia of medicine ${ }^{3,4}$. Modern pharmaceutical studies have demonstrated that $H$. citrina extract has antidepressant, antioxidant, and anti-inflammatory effects $^{5-7}$. The chemical components isolated from $H$. citrina mainly include flavonols, polyphenols, anthraquinones, and alkaloids ${ }^{8}$. Rutin is the main chemical constituent and plays an important role in the antidepressant activity of $H$. citrina ${ }^{5}$; however, the corresponding biosynthetic genes have rarely been reported in this plant. Here we predicted some candidate genes of the rutin biosynthesis pathway by the comparative genomic method. In addition, the relatively fast floral development of $H$. citrina severely restricts the harvest window and places a significant resource strain on post-harvest processing. Moreover, the edible value of $H$. citrina rapidly deteriorates after flowering due to a loss of flavor, leading to substantial food waste. Therefore, it is an urgent task to cultivate new varieties of $H$. citrina with staggered

\section{(c) The Author(s) 2021}

(c) (i) Open Access This article is licensed under a Creative Commons Attribution 4.0 International License, which permits use, sharing, adaptation, distribution and reproduction cc) in any medium or format, as long as you give appropriate credit to the original author(s) and the source, provide a link to the Creative Commons license, and indicate if changes were made. The images or other third party material in this article are included in the article's Creative Commons license, unless indicated otherwise in a credit line to the material. If material is not included in the article's Creative Commons license and your intended use is not permitted by statutory regulation or exceeds the permitted use, you will need to obtain permission directly from the copyright holder. To view a copy of this license, visit http://creativecommons.org/licenses/by/4.0/. 
flowering periods or non-blooming buds via molecular breeding, which could generate tremendous economic value. However, the lack of genomic information restricts the cultivation of new varieties and a high-quality genome of $H$. citrina could provide the possibility of achieving this goal.

The market value of $H$. citrina has been $\sim 1$ billion US dollars for many years. One of the crucial reasons for the limited market value is that colchicine in the flower buds is widely recognized as a poisonous substance ${ }^{1}$. However, the existence of colchicine in $\mathrm{H}$. citrina was questioned by our team several years ago ${ }^{9}$. This study aimed to further determine whether colchicine and its precursors exist in H. citrina or not, based on metabolic data, and to clarify why this alkaloid is not produced according to genomic data. The high-quality and chromosome-level genome of $H$. citrina will provide new insights into the rutin biosynthesis and the lack of colchicine.

\section{Results}

\section{Sequencing and assembly}

We generated $177.52 \mathrm{~Gb}$ of $150 \mathrm{bp}$ paired-end reads and $157.53 \mathrm{~Gb}$ (coverage of $\sim 41.46 \times$ ) of short reads (Supplementary Table S1). Simultaneously, we generated 165 -fold PacBio single-molecule long polymerase reads $(625.85 \mathrm{~Gb}$ with an N50 length of $38.27 \mathrm{~kb}$ ) and 172-fold $\mathrm{Hi}-\mathrm{C}$ data $(646.63 \mathrm{~Gb})$ were used to construct the chromosome-level high-quality reference genome. The genome size was estimated to be $\sim 3.80 \mathrm{~Gb}$ and the heterozygosity rate and repeat sequence contents were $1.28 \%$ and $78.85 \%$, respectively (Supplementary Table S2), based on Illumina resequencing data. In the end, we obtained 3183 contigs with an N50 of $2.08 \mathrm{Mb}$ and a size of $3.77 \mathrm{~Gb}$, which was $\sim 99 \%$ of the estimated size (Table 1). To construct chromosome-level genes, we used $\sim 170 \times \mathrm{Hi}-\mathrm{C}$ data to anchor contigs to chromosomes. We successfully clustered 2919 contigs spanning $3.41 \mathrm{~Gb}(90.36 \%$ of the total length of all contigs) into 11 chromosome groups after further ordering and orienting the clustered contigs (Fig. 1a). Finally, we obtained the first chromosome-level and high-quality genome of $H$. citrina, with chromosome lengths ranging from 216.66 to $471.57 \mathrm{Mb}$, accounting for $90.42 \%$ of the whole sequence (Fig. 1b and Supplementary Table S3).

We first assessed the accuracy and completeness of our assembly results through Benchmarking Universal SingleCopy Orthologs (BUSCO) analysis, then identified 91.4\% complete and 2.4\% partial BUSCO genes (Supplementary Table S4). In addition, $99.49 \%$ of the filtered short reads (157.53 Gb, Supplementary Table S1) were mapped to the genome of $H$. citrina, which covered $99.86 \%$ of the assembly. Furthermore, a total of 22,310 homozygous single-nucleotide polymorphisms (SNPs) $(0.0006 \%$ of the total $H$. citrina assembly) were identified. In summary, the above results demonstrate the high accuracy and completeness of the $H$. citrina genome.

\section{Genome annotation}

Repetitive sequence prediction of the $H$. citrina genome was mainly performed through two methods: homology annotation and $\mathrm{ab}$ initio prediction. A total of $3.25 \mathrm{~Gb}$ of repetitive elements was identified in our assembled genome, comprising $86.20 \%$ of the whole genome (Supplementary Table S5). Among these repetitive elements, long terminal repeats were the main type, accounting for $72.39 \%(2.73 \mathrm{~Gb})$. The rest were short interspersed nuclear elements, DNA transposons, and long interspersed nuclear elements, which accounted for $0.15 \%$, $14.24 \%$, and $6.63 \%$, respectively. Similarly, a total of 3540 transfer RNA (tRNA), 406 ribosomal RNA, 457 small nuclear RNA, and 127 microRNA genes were annotated in the H. citrina genome (Supplementary Table S6).

We predicted 54,295 protein-coding genes in the $H$. citrina genome, with an average length of $8339 \mathrm{bp}$ and an average exon number of 4.53 for each gene (Supplementary Table S7). By comparing the genes annotated in the other six species, we found that the various indicators of the annotated genes (gene, CDS, exon, and intron lengths) were similar to those of other species (Supplementary Fig. S1). We functionally annotated 44,398 (81.77\%)

Table $1 H$. citrina genome assembly results

\begin{tabular}{|c|c|c|c|c|c|}
\hline & Mecat 2 assembly & Post Gcpp & Post Pilon & Haplotig purge & Hi-C assembly \\
\hline Size (Mb) & 5611.82 & 5611.82 & 5611.82 & 3774.13 & 3775.58 \\
\hline No. contigs/Scaffold & 8877 & 8877 & 8877 & 3183 & 734 \\
\hline No. contigs/Scaffold (>2 kb) & 8834 & 8834 & 8842 & 3174 & 725 \\
\hline Max. contig/Scaffold length (bp) & $21,710,810$ & $21,804,269$ & $21,795,804$ & $21,795,804$ & $471,572,209$ \\
\hline Contig/Scaffold N50 size (bp) & $1,516,939$ & $1,522,568$ & $1,521,497$ & $2,081,915$ & $294,951,729$ \\
\hline Contig/Scaffold N90 size (bp) & 428,328 & 430,376 & 428,572 & 761,644 & $216,659,559$ \\
\hline BUSCO & $82.78 \%$ & - & $92.0 \%$ & $91.5 \%$ & $91.2 \%$ \\
\hline
\end{tabular}


$\mathbf{a}$

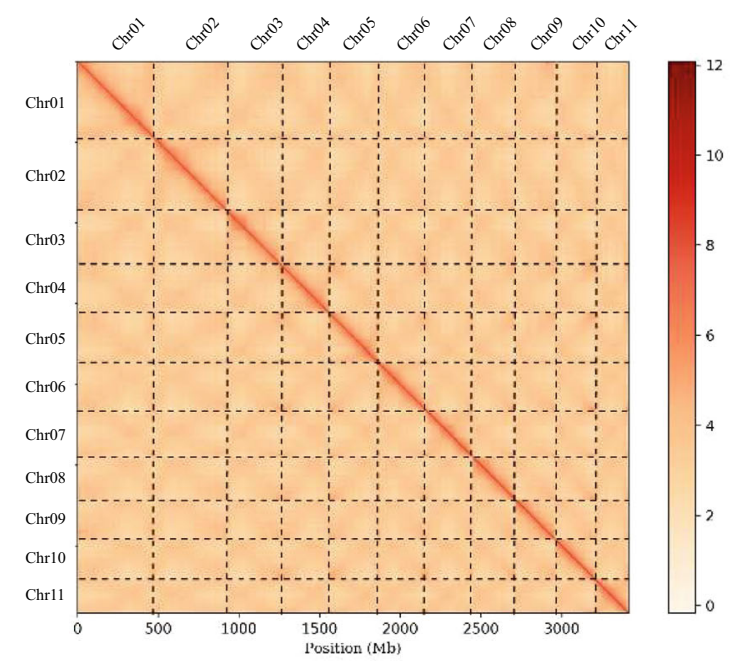

b

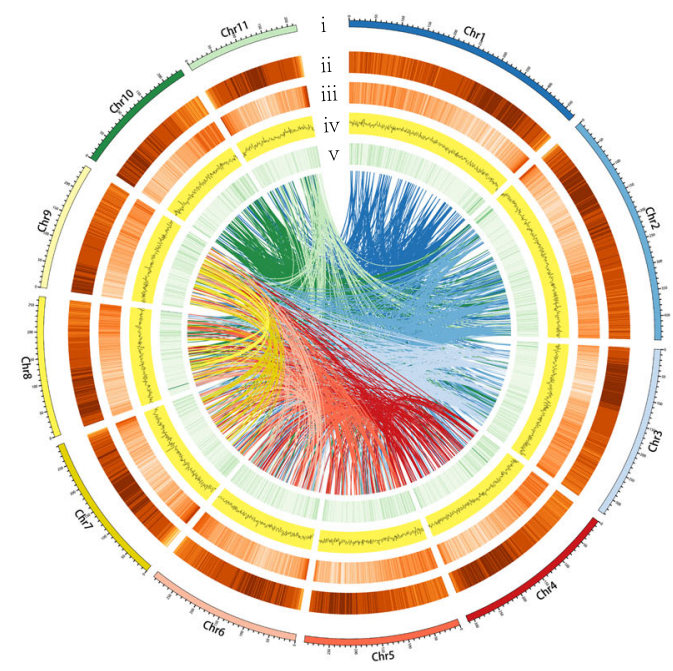

Fig. 1 H. citrina genome assembly. a The Hi-C interaction heatmap for the H. citrina. The color in the figure from light to dark indicates the increase in the intensity of interaction. $\mathbf{b}$ Overview of $\mathrm{H}$. citrina genomic features. (i) Circular representation of the pseudomolecule, (ii-v) repetitive sequence density (bin = $1 \mathrm{M})$, gene density (bin = 1 M), GC content (bin = $1 \mathrm{M})$, ncRNA densities (bin = $1 \mathrm{M})$, and genome collinearity

protein-coding genes of $H$. citrina based on known genes, conserved domains, and Gene Ontology (GO) terms (Supplementary Table S8). Finally, 93.8\% of the BUSCO genes were identified in the annotation of $H$. citrina (Supplementary Table S4), which showed that our annotations were complete and reliable by BUSCO analysis.

\section{Genome evolution and gene families expansion/ contraction}

In this study, we first compared the protein sequences encoded by $H$. citrina with those encoded by 18 other species, namely, Amborella trichopoda, Macleaya cordata, Prunus mume, Arabidopsis thaliana, Theobroma cacao, Camellia sinensis, Rhododendron williamsianum, Solanum tuberosum, Pharbitis nil, Coffee arabica, Chrysanthemum nankingense, Lonicera japonica, Dendrobium catenatum, Phalaenopsis equestris, Asparagus officinalis, Allium sativum, Oryza sativa, and Zea mays. These species had 116 single-copy orthologous gene families according to gene family cluster analysis. In addition, we clustered 51,740 protein sequences $(81.99 \%)$ encoded by $H$. citrina into 15,974 gene families. After length-based filtering of the shared single-copy orthologous gene families, 116 genes remained. The phylogenetic tree showed that the H. citrina, A. sativum, and A. officinalis were located on the same evolutionary branch, showed a closer relationship. In addition, our prediction results showed that $H$. citrina, A. sativum, and A. officinalis phylogenetically diverged from the common ancestor $\sim 71.7 \mathrm{Mya}$, after the separation of Orchidaceae at 107.24 Mya (Fig. 2a), which is consistent with published research ${ }^{10}$.
A total of 42,646 gene families in the most recent common ancestor of the 19 species were obtained by analyzing the gene family expansion and contraction. The number of expanded and contracted gene families in H. citrina were 10,375 and 6707, respectively (Fig. 2a). Compared with A. officinalis and A. sativum, it has 116 expanded and 4591 contracted gene families, which demonstrated that the number of expanded genes in $H$. citrina had increased significantly. This result indicated that $H$. citrina may have experienced more duplication events than A. officinalis and A. sativum. We found that these genes in $H$. citrina were also the most abundant based on the multicopy homologous genes number (Fig. 2b). In addition, we performed GO and KEGG (Kyoto Encyclopedia of Genes and Genomes) enrichment analyses of these expanded and contracted genes in the H. citrina genome. We found lineage-specific expansions of genes related to the metabolic biosynthesis of flavonoids, which may affect the biosynthesis of rutin and enhance flavor and medicinal value (Supplementary Table S9).

\section{Genome-wide duplication events}

To identify the source of many genes $(>50,000)$ in $H$. citrina, we performed whole-genome duplication (WGD) analysis using overlapping $H$. citrina genomes. The synonymous substitution rate (Ks) estimates were applied to detect WGD events. The distribution of Ks values results showed that $H$. citrina have one main peak at Ks values of $\sim 0.18$ ( 15.73 Mya) (Fig. 2c), whereas A. officinalis have more ancient WGD event. Dot plots can be shown as paralogs (2-2 diagonal relationships) 

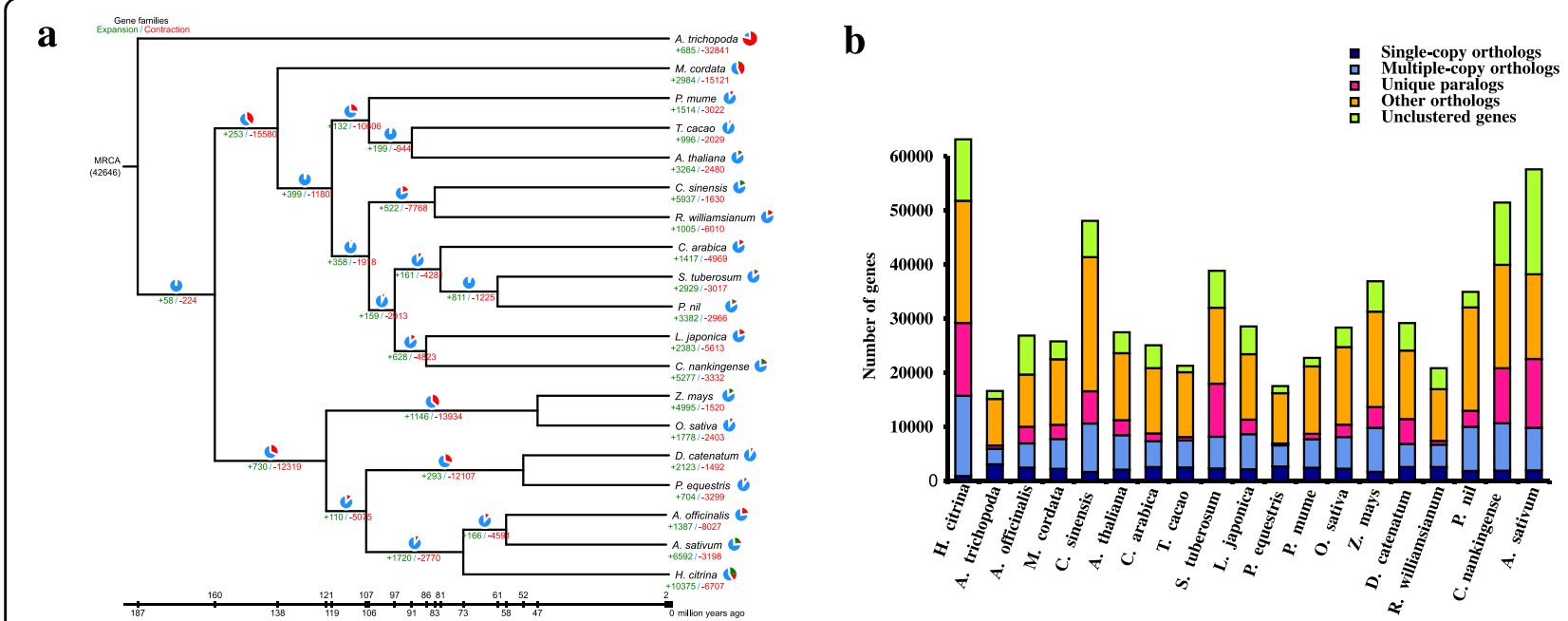

c

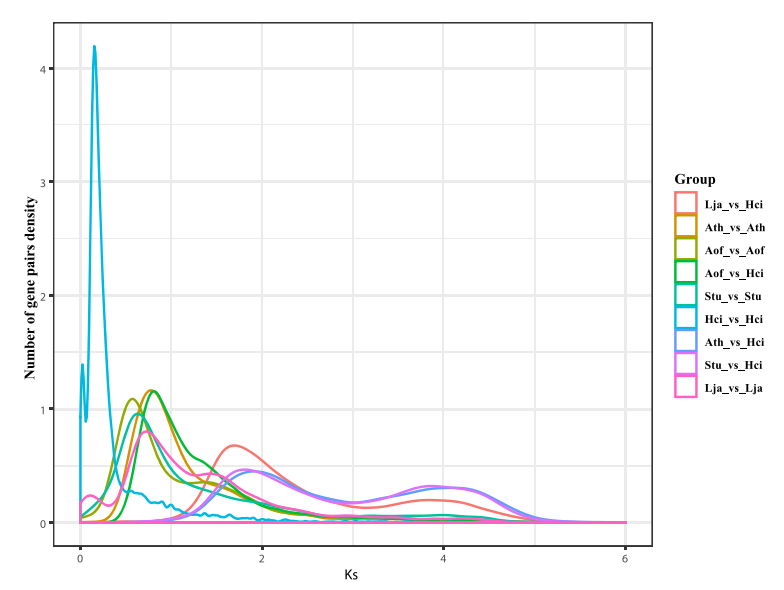

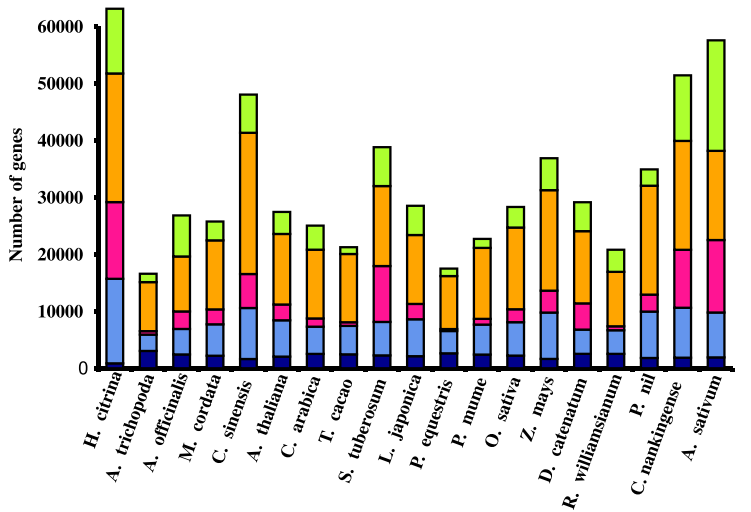

d

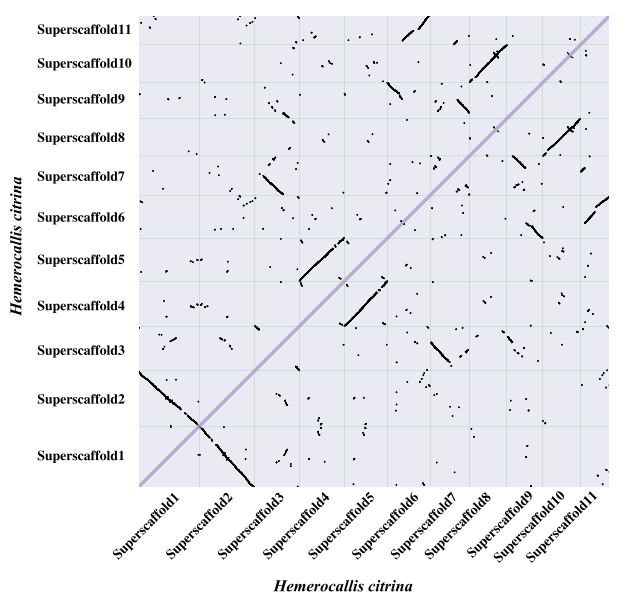

Fig. 2 Evolution of the $\boldsymbol{H}$. citrina genome and gene families. a Phylogenetic tree of 19 plant species and the sizes of expanded and contracted gene families: A. trichopoda, M. cordata, P. mume, T. cacao, A. thaliana, C. sinensis, R. williamsianum, C. arabica, S. tuberosum, P. nil, L. japonica, C. nankingense, Z. mays, O. sativa, D. catenatum, P. equestris, A. officinalis, A. sativum, and H. citrina. b The distribution of single-copy, multiple-copy, unique, other orthologous and unclustered genes in the 19 plant species. c Distribution of Ks values between L. japonica, A. thaliana, A. officinalis, S. tuberosum, and H. citrina (Ks distribution chart showing that $H$. citrina experienced a relatively recent WGD event). $\mathbf{d}$ Dot plots of paralogs in the H. citrina genome

evolving from a recent WGD event in the H. citrina genome (Fig. 2d).

\section{Prediction of rutin biosynthesis genes in $\mathrm{H}$. citrina}

Rutin is the main ingredient and is the recognized one of the main antidepressant compounds in H. citrina. The biosynthetic precursor of rutin is derived from phenylalanine and then synthesized by ten enzymes ${ }^{11}$ (Supplementary Table S10 and Fig. 3a). Through analysis, the expanding and contracting of seven gene families involved in the biosynthesis of rutin. We found four homologous genes (CHS, F3'5' H, FLS, and UGT/ $G T$ ) in $H$. citrina have been increased significantly compared with other species (Fig. 3b). We predicted 108 candidate genes in ten gene families involved in rutin biosynthesis by homologous alignment and a Pfam database search (Fig. 3a). Then, we found that rutin primarily accumulates in the flower buds, whereas the content of rutin in the stems, leaves, and roots is lower according to High-performance liquid chromatography/ quadrupole time-of-flight (HPLC-Q-TOF) methods (Fig. 3c), which indicated that the candidate genes were mainly expressed in flower buds. Finally, 20 candidate genes were predicted in line with this coexpression pattern (Fig. 3a, red color).

\section{Colchicine and its biosynthesis pathway is not existent in H. citrina}

The extracted ion chromatogram (EIC) of the precise $m / z$ value of colchicine standard (Cp 16, $\mathrm{m} / z$ 400.1074, 


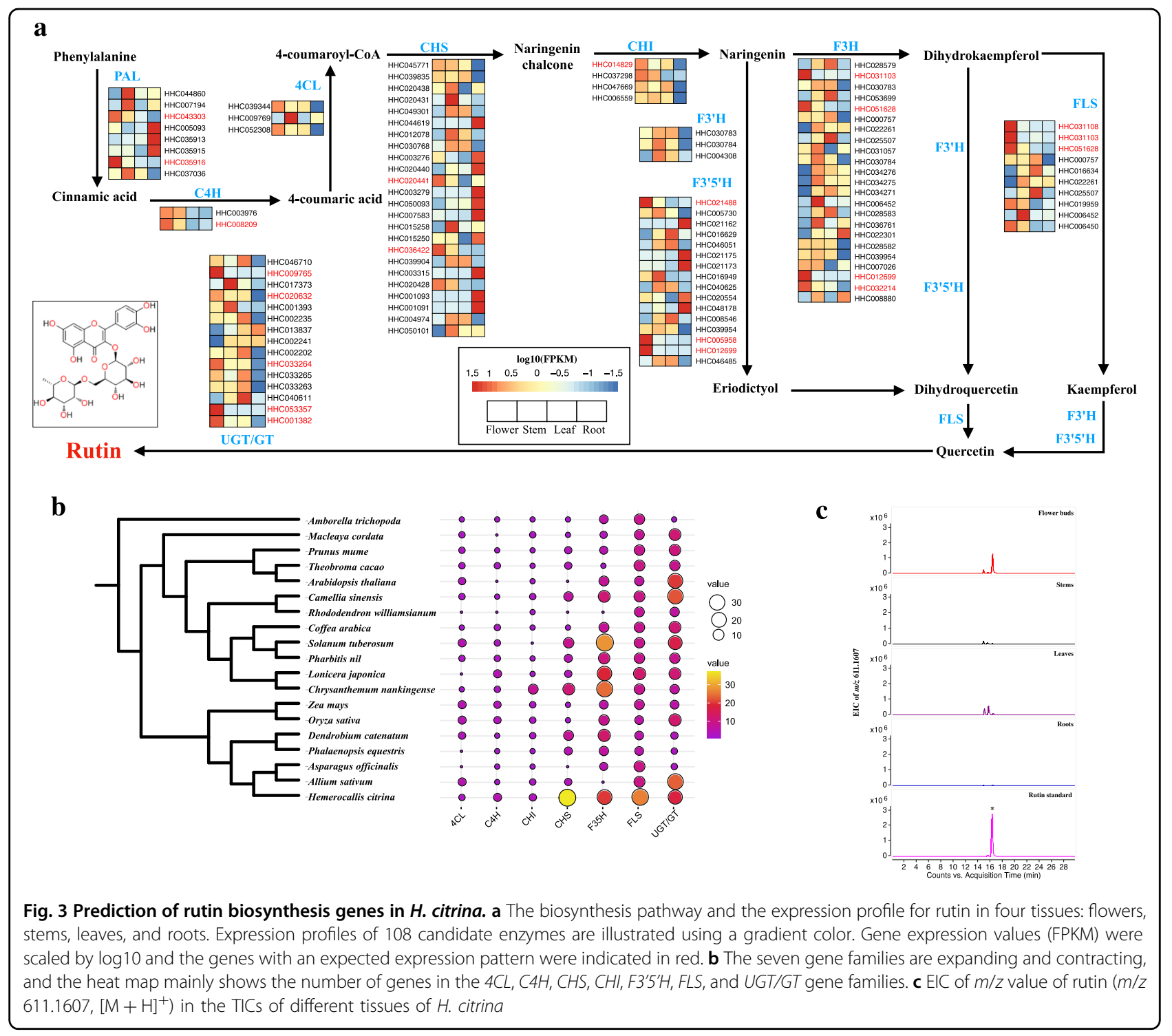

$[\mathrm{M}+\mathrm{H}]^{+}$) for the total ion chromatograms (TICs) of Gloriosa superba, Colchicum autumnale, and H. citrina were performed. Colchicine was found and identified unambiguously in G. superba and C. autumnale by comparing their retention time, precise $m / z$ value, and characteristic fragment ions with those of the standard. However, the precise $m / z$ value of this compound was not found in the TICs of different tissues of $H$. citrina (Fig. $4 \mathrm{a}-\mathrm{c}$ ), which proved that this plant did not contain colchicine.

The near-complete biosynthesis pathway and related functional genes of colchicine have been identified in G. superba ${ }^{12}$ (Fig. 4d). The EIC of the theoretical $\mathrm{m} / \mathrm{z}$ values of 15 compounds ( $\mathrm{Cp} 1$ to $\mathrm{Cp} \mathbf{1 5}$ ), which were the precursors of colchicine, were performed for the TICs of G. superba, C. autumnale, and H. citrina. These precursors were detected and determined from G. superba and C. autumnale by their precise $m / z$ values and characteristic fragment ions. However, only two original amino acids, L-tyrosine ( $\left(\begin{array}{ll}\mathrm{P} & \mathbf{1}\end{array}\right)$ and L-phenylalanine (Cp 3), were observed and identified in H. citrina (Supplementary Figs. S2 and S4), and the theoretical $\mathrm{m} / z$ values of the remaining 13 precursors were not found (Supplementary Figs. S3 and S5-S16). In addition, the candidate genes involved in the colchicine biosynthesis pathways were detected by BLASTP searching with eight known genes from G. superba ${ }^{12}$ (with an $E$-value $\leq 1 \mathrm{e}-5$, a coverage $\geq 0.5$, and an identity $\geq 0.5$ ) (Fig. 4d and Supplementary Table S11). Unsurprisingly, none of the orthologous genes were obtained from $H$. citrina. Therefore, the genomic analysis demonstrated that $H$. citrina does not contain any genes involved in colchicine biosynthesis. 


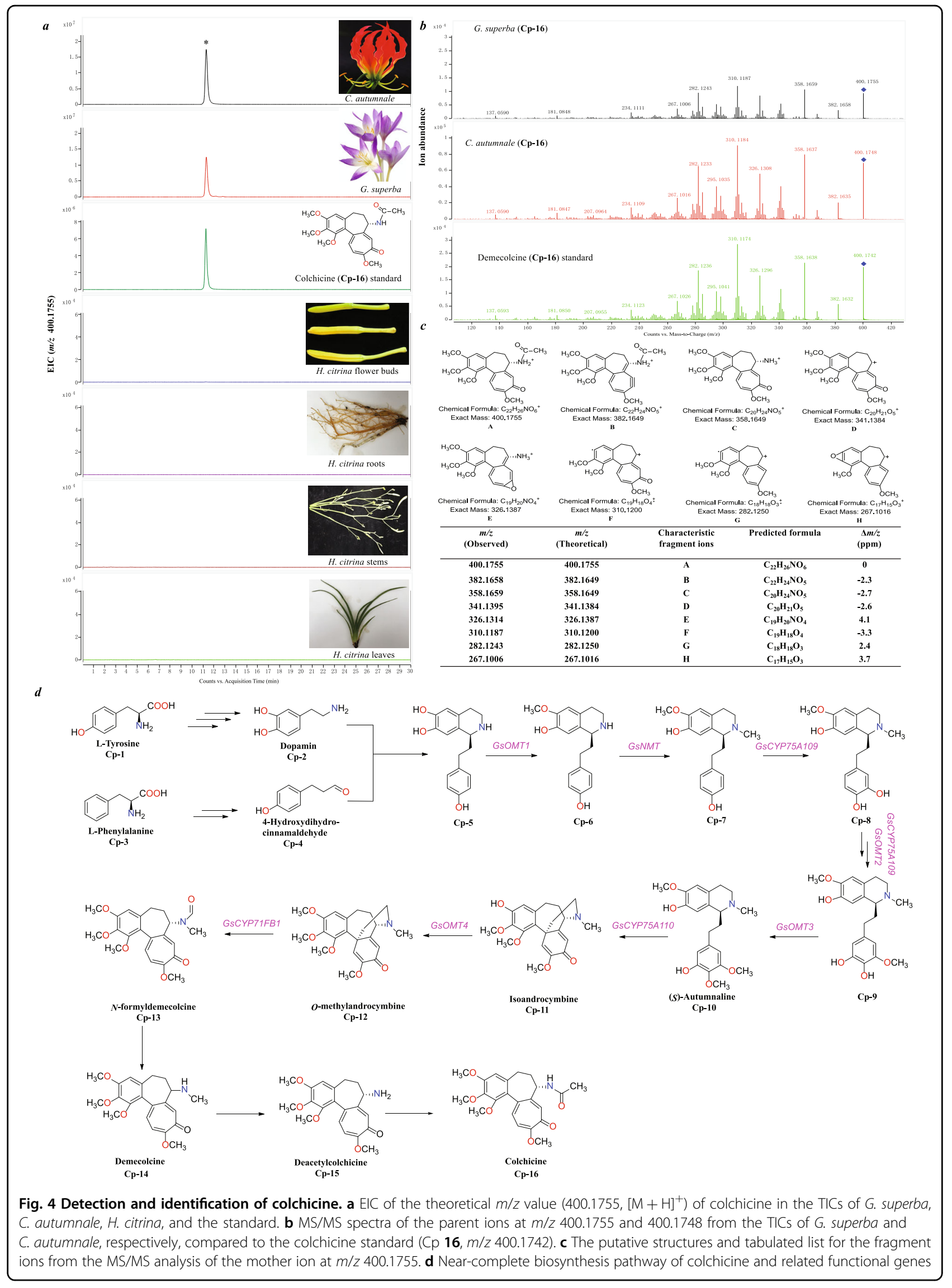




\section{Discussion}

We construct a high-quality and chromosome-level reference genome by combining PacBio SMRT and Hi-C technology. We found that the genome data of $H$. citrina have high heterozygosity and repetitive content features. More importantly, we can use the genome to research the phylogenetic and evolutionary characteristics at a deeper level, and to cultivate new varieties of $H$. citrina with staggered flowering periods or non-blooming buds via molecular breeding. Based on the multi-omics analysis, we deduced a gene coexpression rule and predicted that 20 candidate genes match this rule. These results lay the groundwork for further research on the functional genes involved in the biosynthesis pathway of rutin.

Numerous journals, magazines, newspapers, and other news outlets have reported that $H$. citrina contains colchicine, which was first identified in Hemerocallis by microchemical methods in $1929^{13}$; however, the identification method and result were doubted by other scientists in $1949^{14}$. In 1977, colchicine was first reported from $H$. citrina in China ${ }^{15}$. In the next few decades, more than 30 poisoning incidents were recorded in China due to the consumption of the fresh flower buds of $H$. citrina, which resulted to more than 830 people with symptoms of poisoning. All reports stated that the poisoning was caused by colchicine in $H$. citrina (Supplementary Table S12). Moreover, $H$. citrina containing colchicine was even recorded in college textbooks and popular science books in China ${ }^{16-19}$. However, this compound was not found in different tissues of $H$. citrina using HPLC-Q-TOF-mass spectrometry (MS) technologies in this study. In addition, none of the orthologous genes involved in the colchicine biosynthesis pathway were identified in the $H$. citrina genome, which further clarified that this alkaloid was absent at the genomic level. Both results unambiguously demonstrate that $H$. citrina does not contain colchicine. In past studies, colchicine was never isolated and identified from $H$. citrina by phytochemical methods. In addition, this alkaloid was only determined by thin-layer chromatography or HPLC by comparing the Rf value or the retention time (Rt) with that of the standard ${ }^{20,21}$, so another compound $(\mathrm{m} / \mathrm{z} 455.1455$ in positive mode) had $\mathrm{Rf}$ and $\mathrm{Rt}$ values close to those of colchicine $(\mathrm{m} / \mathrm{z}$ 400.1755 in positive mode). Therefore, this compound was incorrectly identified as colchicine ${ }^{9}$. This study challenges the long-standing belief that colchicine present in $H$. citrina leads to poisoning.

\section{Conclusion}

Here, a high-quality and chromosome-scale $H$. citrina genome was reported. The genome was $\sim 3.8 \mathrm{~Gb}$ in size, with a heterozygosity rate of $\sim 1.28 \%$ and contig N50 of $2.09 \mathrm{Mb}$. Subsequently, $\mathrm{Hi}-\mathrm{C}$ technology was applied and we anchored $90.42 \%$ of the assembled contigs to 11 pseudochromosomes. We identified a total of 54,295 protein-coding genes and 63,105 transcripts. Based on comparative genomics, we found that $H$. citrina experienced a recent WGD event at $\sim 15.73$ Mya that increased the number of genes by more than 50 thousand and expanded gene families by more than 10 thousand. A total of 4 gene families involved in the rutin biosynthesis pathway were expanded and 20 candidate genes were predicted by multi-omic data. Finally, we proved for the first time that the biosynthesis pathway of colchicine does not exist in the genome of $H$. citrina. Our research provided the first chromosome-level genome of the Hemerocallis genus, which laid the foundation for genetic research and molecular breeding of $H$. citrina.

\section{Materials and methods}

\section{Sample collection and high-throughput sequencing}

The $H$. citrina was cultivated at Hunan Agricultural University. We collected the healthy leaves from the bestgrowing $H$. citrina. A modified cetyltrimethyl ammonium bromide (CTAB) method $^{22}$ was used for DNA extraction. RNA contaminants were removed by RNase A and the integrity of DNA was obtained. The DNA molecules were used to construct a library after being cut into $\sim 30 \mathrm{~kb}$ fragments and then sequenced on the PacBio Sequel II platform (Frasergen, China). Simultaneously, a library with an insert size of $350 \mathrm{bp}$ was constructed for the Illumina HiSeq X Ten platform (Illumina, Inc., San Diego, CA, USA). These short reads for whole-genome sequencing were mainly used for genome survey, error correction, and polishing after initial assembly. A Hi-C library was established using the young leaves of $H$. citrina and the BGI MGISEQ-2000 platform (BGI, China) was used for sequencing. In addition, the size of $H$. citrina genome was evaluated by $k$-mer analysis with GCE $^{23}$ (Supplementary Fig. S17).

\section{RNA extraction and Iso-Seq sequencing}

H. citrina was grown in Qidong County (Hunan, China, coordinates: $111^{\circ} 52^{\prime} 22.44^{\prime \prime} \mathrm{E}, 26^{\circ} 53^{\prime} 23.75^{\prime \prime} \mathrm{N}$ ) for RNA extraction. We sampled fresh, healthy roots, stems, leaves, and flowers from five different periods with three biological duplication. We used TRIzol reagent (Invitrogen, USA) to extract total RNA based on the recommended protocol. DNA was removed via RQ1 DNase (Promega, USA). Finally, RNA from all samples was mixed to construct the library.

The cDNA synthesis kit (ClontechSMARTer®) was used to establish the cDNA libraries. AMPure PB beads were employed for the cDNA product purification. A total of $376.06 \mathrm{Mb}$ was sequenced with $30 \mathrm{~h}$ movies by PacBio Sequel II platform (Supplementary Table S1). Simultaneously, these RNAs were used to construct shortfragment libraries and then processed on the BGI 
platform, which yielded $30.74 \mathrm{~Gb}$ of raw RNA sequence data with a read quality Q30 of $91.0 \%$.

\section{Genome assembly}

All subread data from SMRT sequencing were used for $H$. citrina genome assembly. The draft genome assembly was obtained using mecat $2(20,190,226)$ with the default parameters. The gcpp in the SMRT link 4 toolkit was performed to correct errors after the initial assembly of the genome. Then, we used $157.53 \mathrm{~Gb}$ of short reads to correct any remaining errors with Pilon $^{24}$ (v1.22). Due to the heterozygosity of the genome, Haplotigs purge was used to filter redundant sequences ${ }^{25}$.

Pseudochromosomes were determined using $\mathrm{Hi}-\mathrm{C}$ analysis, as described previously ${ }^{26}$. Briefly, $646.63 \mathrm{~Gb}$ of clean read pairs were produced from the Hi-C library and mapped to the polished $H$. citrina contig assembly using BWA (bwa-0.7.17) with the default parameters ${ }^{27}$. LACHESIS $^{28}$ tool was used to cluster contigs into chromosome-level scaffolds by the genomic proximity signal of $\mathrm{Hi}-\mathrm{C}$ data.

\section{Evaluation of genome quality}

Genome assembly accuracy and completeness were first assessed using the continuous long reads subreads. A total of $96.60 \%$ of subreads were mapped to $99.97 \%$ of the genome, with an average depth of $129.89 \times$. Then, a window of size $10 \mathrm{~kb}$ was used to continuously slide along the genome without overlapping (when the sequence length was $<10 \mathrm{~kb}$, the actual length prevailed), calculate the average sequencing depth of the sequence in the window and the percentage of GC content. Finally, draw the contig GC content distribution-sequencing depth distribution density map based on the statistical data (Supplementary Fig. S18). Second, the single-base level genome assembly was evaluated using Illumina short-read by BWA 0.7.17 software ${ }^{27}$. Furthermore, homozygous SNPs were filtered by the GATK 4.0.8.1 ${ }^{29}$ package. The assembled genome was also subjected to BUSCO v3.0.2 30 analysis with embryophyta_odb10 to evaluate the completeness of the genome and annotation.

\section{Annotation of repetitive sequences and genes}

De novo and homology-based prediction methods were employed to annotate the repeat sequences in the genome of $H$. citrina. The known transposable elements within the $H$. citrina genome were identified by combining RepeatMasker $^{31}$, RepeatProteinMask, and RepeatModeler. In addition, the tRNA-related genes were mainly identified by tRNAscan-SE $(\mathrm{v} 1.3 .1)^{32}$ and Infernal $(\mathrm{v} 1.1 .2)^{33}$ software with default parameters.

The assembled genome of $H$. citrina was hard and soft masked by RepeatMasker prior to gene prediction. First, we used homologous proteins to train the gene models of
Augustus (v3.3.1) $)^{34}$ and $\mathrm{SNAP}^{35}$, and then performed ab initio gene prediction based on these models. Second, the protein sequences were predicted genes using Exonerate $(\mathrm{v} 2.2 .0)^{36}$ with the default parameters. Third, the clean RNA-Sequencing reads were assembled into transcripts via Trinity ${ }^{37}$ to perform RNA-based gene prediction and the gene structure was further predicted using $\mathrm{PASA}^{38}$. Finally, Maker (v3.00) $)^{39}$ was employed to integrate the prediction results of the three strategies.

Gene functions were inferred by aligning our annotated gene models with known databases. BLAST $+(\mathrm{v} 2.6 .0+)^{40}$ was performed against the National Center for Biotechnology Information (NCBI), Non-Redundant, TrEMBL, and Swiss-Prot ${ }^{41}$. The protein domains were annotated using PfamScan ${ }^{42}$ and InterProScan $(\mathrm{v} 5.35-74.0)^{43}$ based on InterPro protein databases. The motifs and domains were identified by $\mathrm{Pfam}^{44}$. GO ${ }^{45}$ IDs for each gene were obtained from Blast2GO ${ }^{46}$. KEGG Automatic Annotation Server was used to annotate the KEGG pathways ${ }^{47}$.

\section{Gene family identification}

To cluster families of protein-coding genes, proteins from the longest transcripts of each gene from $H$. citrina and other closely related species, including $A$. trichopoda, M. cordata, P. mume, A. thaliana, T. cacao, C. sinensis, R. williamsianum, S. tuberosum, P. nil, C. arabica, C. nankingense, $L$. japonica, D. catenatum, P. equestris, A. officinalis, A. sativum, O. sativa, and $Z$. mays, were used. All proteins were extracted and aligned with each other using BLASTP ${ }^{40}$ programs (NCBI blast v2.6.0) with a maximal $E$-value of $1 e-5$. We filtered out and excluded putative fragmented genes with an identity $<30 \%$, a coverage $<50 \%$, and protein-encoding sequences shorter than $50 \mathrm{bp}$. Then, we used OrthoMCL (v14-137) ${ }^{48}$ to cluster genes from different species into gene families.

\section{Phylogenetic analysis}

We construct a phylogenetic tree of $H$. citrina and other closely related species by the protein sequences of 186 single-copy orthologous genes, which were aligned with the MUSCLE (v3.8.31) ${ }^{49}$ program, and we further employed RAxML (v8.2.11) ${ }^{50}$ to build the phylogenetic tree.

\section{Gene families expansion/contraction}

According to the identified gene families and the constructed phylogenetic tree with the predicted divergence times of those species, we used CAFÉ ${ }^{51}$ to analyze gene families expansion and contraction. Families with a $p$-value $<$ 0.05 were considered to have an accelerated rate of gene gain or loss. These gene families in H. citrina ( $p$-value $\leq 0.05$ ) were mapped to KEGG pathways for functional enrichment analysis, which was conducted using enrichment methods. For this process, hypergeometric test algorithms were 
implemented and the $Q$-value (false discovery rate) was calculated to adjust $p$-values utilizing the $\mathrm{R}$ environment (https://github.com/StoreyLab/qvalue).

\section{Whole-genome duplication analysis}

We used the synonymous substitution rate (Ks) to detect WGD events. First, syntenic paralogous blocks were identified with MCSCAN between $L$. japonica, $A$. thaliana, A. officinalis, S. tuberosum, and H. citrina. Then, the protein sequences of these plants in the syntenic paralogous blocks were aligned against each other with Blastp $(E$-value $\leq 1 e-5)$ to identify the conserved paralogs of each plant. Third, the Ks values of these gene pairs were calculated. Finally, the Ks distribution was used to evaluate the WGD events.

\section{Sample collection and preparation for metabolomic analysis}

G. superba, C. autumnale, and $H$. citrina plants were collected from Kunming University of Science and Technology, China Pharmaceutical University, and Hunan Agriculture University, respectively. All samples (whole G. superba and C. autumnale, and flower buds, roots, stems, and leaves of $H$. citrina) were freeze-dried and crushed by a disintegrator. Approximately $0.4 \mathrm{~g}$ of powdered sample was extracted using ultrasonic bath for $120 \mathrm{~min}$ with $10 \mathrm{~mL}$ of $70 \%$ methanol-water $(\mathrm{v} / \mathrm{v})$. The extract solution was filtered by a $0.22 \mu \mathrm{m}$ microporous membrane and stored in a bottle.

\section{HPLC-Q-TOF-MS conditions}

HPLC-Q-TOF-MS conditions were optimized based on the previous method ${ }^{1}$. The gradient of elution was modified as follows: $0-3 \mathrm{~min}, 10-15 \%$ (B); $3-8 \mathrm{~min}, 15-30 \%$ (B); 8-16 min, $30-65 \%$ (B); and 16-30 min, 65-95\% (B). The injection volume was reduced to $2 \mu \mathrm{L}$ and the MS/ MS data of each compound were obtained using different collision energy $(10-35 \mathrm{eV})$.

\section{Acknowledgements}

This work was supported by the "National Key R\&D Program of China (2017YFD0501500)," "Hunan Provincial Key Research and Development Project (2020NK2031)," and "The Special Funds for Development of Local Science and Technology from Central Government (2019XF5067)."

\footnotetext{
Author details

${ }^{1}$ Hunan Key Laboratory of Traditional Chinese Veterinary Medicine, Hunan Agricultural University, Changsha, Hunan 410128, China. ${ }^{2}$ College of Veterinary Medicine, Hunan Agricultural University, Changsha, Hunan 410128, China. ${ }^{3}$ Wuhan Frasergen Bioinformatics Co., Ltd, Wuhan, Hubei 430075, China. ${ }^{4}$ College of Animal Science and Technology, Hunan Agricultural University, Changsha, Hunan 410125, China. ${ }^{5}$ Green Melody Bio-engineering Group Company Limited, Changsha, Hunan 410329, China. ${ }^{6}$ National and Local Union Engineering Research Center of Veterinary Herbal Medicine Resource and Initiative, Hunan Agricultural University, Changsha 410128, China
}

\section{Author contributions}

Z.Q., P.H., and J.Z. conceived and designed the study. Z.Q., J. Liu, and P.H. collected the sample. X.Y., G.H., and J. Lao estimated the genome size and assembled the genome. X.Z. and P.H. performed DNA, RNA-sequencing, and $\mathrm{Hi}-\mathrm{C}$ experiments. M.S. and P.H. performed the genome annotation and functional genomic analysis. Z.Q., X.L., and Z.Y. performed the data analysis of metabolome. Z.Q., X.Y., and P.H. wrote the manuscript.

\section{Data availability}

All sequencing data were deposited in the NCBI Sequence Read Archive (SRA) database with BioProject accession number PRJNA647253. The assembled genome was submitted to DDBJ/ENA/GenBank with accession number JACEHZ000000000. The version is JACEHZ010000000.

\section{Conflict of interest}

The authors declare no competing interests.

Supplementary information The online version contains supplementary material available at https://doi.org/10.1038/s41438-021-00539-6.

Received: 30 December 2020 Revised: 19 March 2021 Accepted: 26 March 2021

Published online: 07 April 2021

\section{References}

1. Liu, J. et al. Systematic identification metabolites of Hemerocallis citrina Borani by high-performance liquid chromatography/quadrupole-time-of-flight mass spectrometry combined with a screening method. J. Pharm. Biomed. Anal. 186, 113314 (2020)

2. $\mathrm{Ma}, \mathrm{G}$. et al. iTRAQ-based quantitative proteomic analysis reveals dynamic changes during daylily flower senescence. Planta 248, 859-873 (2018).

3. Li, C. F. et al. Evaluation of the toxicological properties and anti-inflammatory mechanism of Hemerocallis citrina in LPS-induced depressive-like mice. Biomed. Pharmacother. 91, 167-173 (2017).

4. Yang, R. F., Geng, L. L., Lu, H. Q. \& Fan, X. D. Ultrasound-synergized electrostatic field extraction of total flavonoids from Hemerocallis citrina baroni. Ultrason. Sonochem. 34, 571-579 (2017).

5. Lin, S. H. et al. The antidepressant-like effect of ethanol extract of daylily flowers (Jinn Zhēn Huā) in rats. J. Tradit. Complement. Med. 3, 53-61 (2013).

6. Wang, J. et al. Ethyl acetate fraction of Hemerocallis citrina Baroni decreases tert-butyl hydroperoxide-induced oxidative stress damage in BRL-3A cells. Oxid. Med. Cell Longev. 2018, 1-13 (2018).

7. Tian, $\mathrm{H}$. et al. Effects of phenolic constituents of daylily flowers on corticosterone-and glutamate-treated PC12 cells. BMC Complement. Alter. Med. 17, 69 (2017).

8. Xu, P. et al. Antidepressant-like effects and cognitive enhancement of the total phenols extract of Hemerocallis citrina Baroni in chronic unpredictable mild stress rats and its related mechanism. J. Ethnopharmacol. 194, 819-826 (2016).

9. Tang, M. N., Liu, X. B., Huang, J. L., Deng, F. M. \& Zeng, J. G. Questioning and arguable research on edible Hemerocallis citrina containing colchicine. Chin. Tradit. Herb. Drugs 047, 3293-3300 (2016).

10. Li, S. F. et al. Chromosome-level genome assembly, annotation and evolutionary analysis of the ornamental plant Asparagus setaceus. Hortic. Res. 7, 48 (2020).

11. Zhang, L. et al. The tartary buckwheat genome provides insights into rutin biosynthesis and abiotic stress tolerance. Mol. Plant. 10, 1224-1237 (2017).

12. Nett, R. S., Lau, W. \& Sattely, E. S. Discovery and engineering of colchicine alkaloid biosynthesis. Nature 584, 148-153 (2020).

13. Klein, G. \& Soos, G. Der mikrochemische Nachweis der Alkaloide in der Pflanze Oesterr Bot. Z. 78, 157-163 (1929).

14. Traub, H. P. Colchicine poisoning in relation to Hemerocallis and some other plants. Science 110, 686-687 (1949).

15. Li, Z. H. Plant poisoning. Barefoot Dr. Mag. 8, 44-45 (1977).

16. Zong, W., Zhang, L. \& Wang, M. Z. (eds) Food Safety (Chemical Industry, 2016).

17. Zhang, Z. J. et al. (eds) Introduction of Food Safety (Chemical Industry, 2015).

18. Peng, W. X., Pan, T., Yuan, Y. Y. \& Wang, L. (eds) Food Safety and Food Poisoning-First Aid Knowledge (GuiZhou, 2012). 
19. Zhou, C. Q. et al. (eds) Food Nutrition (China Metrology, 2006).

20. Hong, Y. F., Cheng, Z. W., Li, J. H. \& Hu, C. On different methods to treat the fresh Hemerocallis citrina and lead to the change of colchicine. J. Hunan Agric. Univ. 29, 500-502 (2003).

21. Zhang, N. et al. Optimization of HPLC detection system for colchicine content in flower buds of Hemerocallis. J. Agric. Univ. Hebei. 9, 48-54 (2017).

22. Doyle, J. J. \& Doyle, J. L. A rapid DNA isolation procedure for small quantities of fresh leaf tissue. Phytochem. Bull. 19, 11-15 (1987).

23. Marçais, G. \& Kingsford, C. A fast, lock-free approach for efficient parallel counting of occurrences of k-mers. Bioinformatics 27, 764-770 (2011).

24. Walker, B. J. et al. Pilon: an integrated tool for comprehensive microbial variant detection and genome assembly improvement. PLOS ONE 9, e112963 (2014).

25. Roach, M. J., Schmidt, S. \& Borneman, A. R. Purge Haplotigs: synteny reduction for third-gen diploid genome assemblies. BMC Bioinformatics 19, 460 (2018).

26. Yin, D. et al. Genome of an allotetraploid wild peanut Arachis monticola: a de novo assembly. Gigascience 7, giy066 (2018).

27. Li, H. Aligning sequence reads, clone sequences and assembly contigs with BWA-MEM. Preprint at arXiv:1303.3997 (2013).

28. Burton, J. N. et al. Chromosome-scale scaffolding of de novo genome assemblies based on chromatin interactions. Nat. Biotechnol. 31, 1119-1125 (2013).

29. McKenna, A. et al. The Genome Analysis Toolkit: a MapReduce framework for analyzing next-generation DNA sequencing data. Genome Res. 20, 1297-1303 (2010).

30. Simão, F. A. et al. BUSCO: assessing genome assembly and annotation completeness with single-copy orthologs. Bioinformatics 31, 3210-3212 (2015).

31. Tarailo-Graovac, M. \& Chen, N. Using repeatMasker to identify repetitive elements in genomic gequences. Curr. Protoc. Bioinformatics 25, 4.10.1-4.10.14 (2004).

32. Lowe, T. M. \& Eddy, S. R. tRNAscan-SE: a program for improved detection of transfer RNA genes in genomic sequence. Nucleic Acids Res. 25, 955-964 (1997).

33. Nawrocki, E. P., Kolbe, D. L. \& Eddy, S. R. Infernal 1.0: inference of RNA alignments. Bioinformatics 25, 1335-1337 (2009).

34. Stanke, M., Keller, O., Gunduz, I., Hayes, A., Waack, S. \& Morgenstern, B. AUGUSTUS: ab initio prediction of alternative transcripts. Nucleic Acids Res. 34, 435-439 (2006).
35. Korf, I. Gene finding in novel genomes. BMC Bioinformatics 5, 59 (2004).

36. Slater, G. S. C. \& Birney, E. Automated generation of heuristics for biological sequence comparison. BMC Bioinformatics 6, 31 (2005).

37. Grabherr, M. G. et al. Full-length transcriptome assembly from RNA-Seq data without a reference genome. Nat. Biotechnol. 29, 644-652 (2011).

38. Haas, B. J. et al. Improving the Arabidopsis genome annotation using maximal transcript alignment assemblies. Nucleic Acids Res. 31, 5654-5666 (2003).

39. Cantarel, B. L. et al. MAKER: an easy-to-use annotation pipeline designed for emerging model organism genomes. Genome Res. 18, 188-196 (2008).

40. Camacho, C. et al. BLAST plus: architecture and applications. BMC Bioinformatics. BioMed. Cent. 10, 1 (2009).

41. Boeckmann, B. et al. The SWISS-PROT protein knowledgebase and its supplement TrEMBL in 2003. Nucleic Acids Res. 31, 365-370 (2003).

42. Mistry, J., Bateman, A. \& Finn, R. D. Predicting active site residue annotations in the Pfam database. BMC Bioinformatics 8, 298 (2007).

43. Jones, P. et al. InterProScan 5: genome-scale protein function classification. Bioinformatics 30, 1236-1240 (2014).

44. Hotz, G. C., Forslund, K., Eddy, S. R., Sonnhammer, E. L. \& Bateman, A. The Pfam protein families database. Nucleic Acids Res. 36, S281-S288 (2008).

45. Ashburner, M. et al. Gene ontology: tool for the unification of biology. The Gene Ontology Consortium. Nat. Genet. 25, 25-29 (2000).

46. Conesa, A. \& Götz, S. Blast2GO: a comprehensive suite for functional analysis in plant genomics. Int. J. Plant Genomics. 2008, 619832 (2008).

47. Kanehisa, M., Goto, S., Sato, Y., Furumichi, M. \& Tanabe, M. KEGG for integration and interpretation of large-scale molecular data sets. Nucleic Acids Res. 40, D109-D114 (2012).

48. Li, L., Stoeckert, C. J. \& Roos, D. S. OrthoMCL: identification of ortholog groups for eukaryotic genomes. Genome Res. 13, 2178-2189 (2003).

49. Edgar, R. C. MUSCLE: multiple sequence alignment with high accuracy and high throughput. Nucleic Acids Res. 32, 1792-1797 (2004).

50. Stamatakis, A. RAxML version 8: a tool for phylogenetic analysis and post-analysis of large phylogenies. Bioinformatics 30, 1312-1313 (2014).

51. Han, M. V., Thomas, G. W., Lugo-Martinez, J. \& Hahn, M. W. Estimating gene gain and loss rates in the presence of error in genome assembly and annotation using CAFE 3. Mol. Biol. Evol. 30, 1987-1997 (2013). 